\title{
The Effects of Bilateral Motor Training on the Power of Grip in Affected Hand of Children with Spastic Hemiplegic Cerebral Palsy
}

\section{Nashmin Azizi Darabkhani, Ali Heyrani*}

Department of Sports Sciences, Faculty of Sports Sciences, Razi University, Kermanshah, Iran

\section{ABSTRACT}

Introduction: This study aimed to examine the effects of six weeks of bilateral motor trainings in the mirror on the power of grip in affected hands in children with spastic hemiplegic cerebral palsy. Materials and Methods: In this clinical trial study, 16 children with hemiplegic cerebral palsy were divided into two groups of bilateral motor exercises with and without mirror. In addition to the usual rehabilitation program, both groups performed motor exercises according to their instructions. The grip strength of the affected hand in both groups was assessed using a digital dynamometer pre and post intervention. Results: The results showed that both groups had a significant improvement in grip strength scores of the affected hand after six weeks of intervention. Also, the between group comparisons showed that the mirror training group was better than other group in the above -mentioned variable. Conclusion: The pattern of these findings suggests that despite the effect of bilateral motor exercises on the grip strength of children with spastic hemiplegic cerebral palsy, bilateral motor exercises with a mirror had a greater effect, which can be explained by the mechanism of mirror neurons.

*Corresponding Author: Ali Heyrani

Email: ali.heyrani@gmail.com 


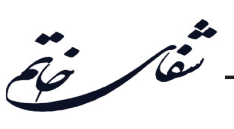

\section{تأثير تمرين حركتى دو طرفه بر قدرت جنَش دست مبتلا در كودكان فلج مغزى همى یلزيك

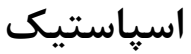

\section{نشمين عزيزى دار ابخانى، على حيرانى"}

كروه علوم ورزشى، دانشكده علوم ورزشى، دانشكاه رازى، كرمانشاه، ايران

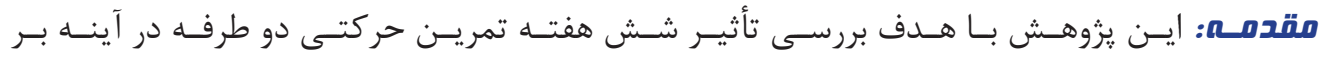

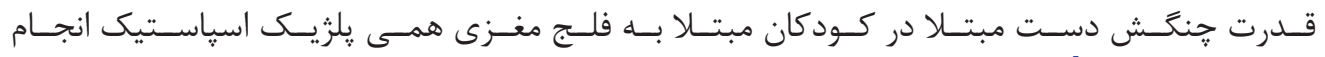

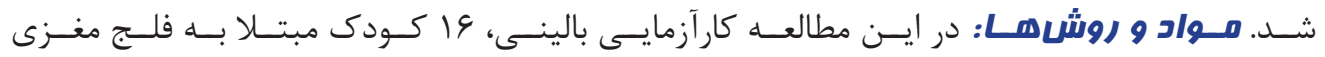

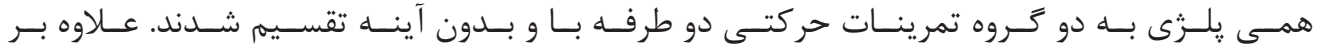

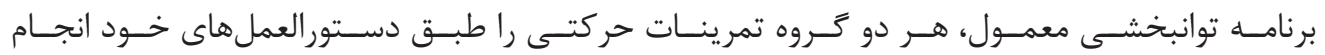

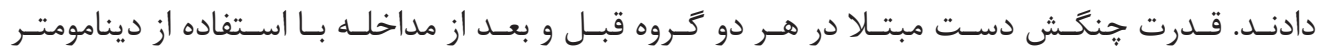

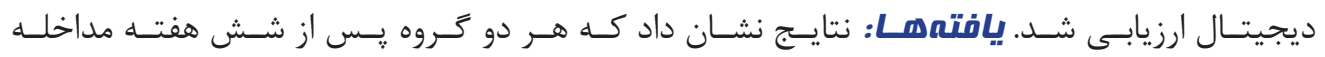

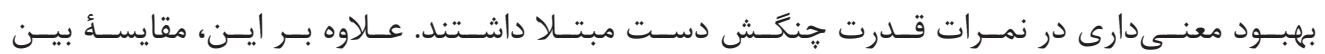

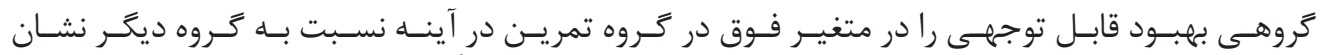

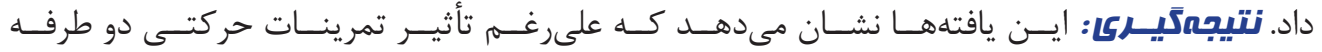

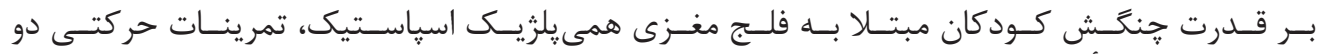

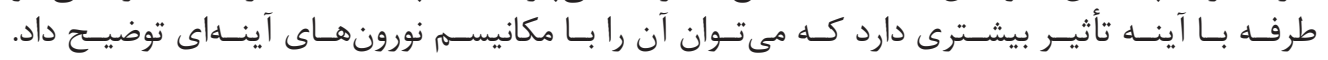




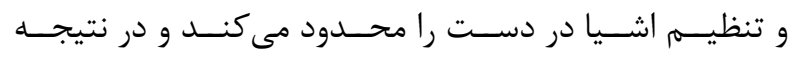
مقدمه

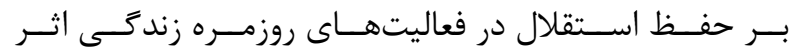

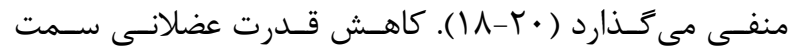

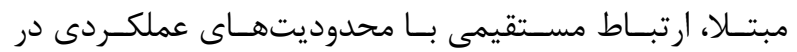

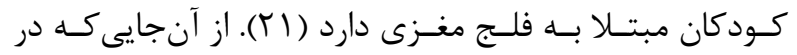

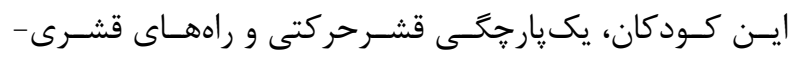

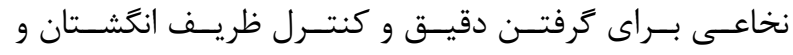

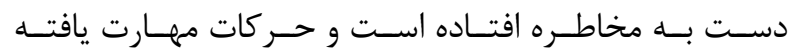

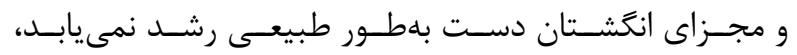

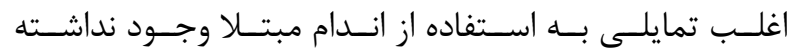

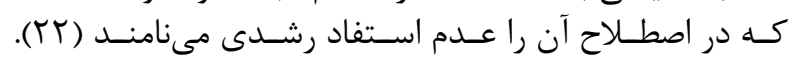

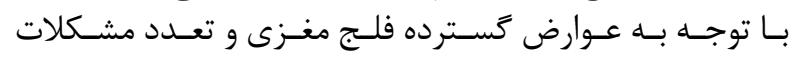

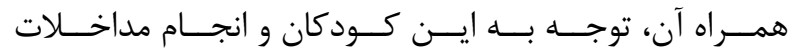

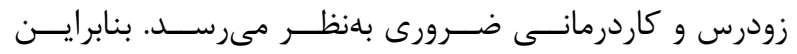

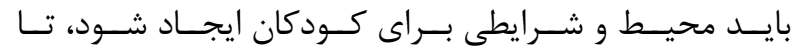

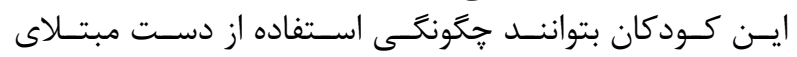

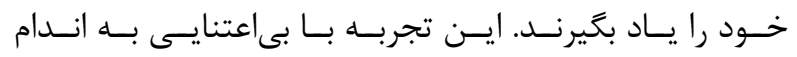

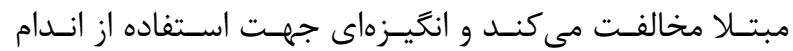

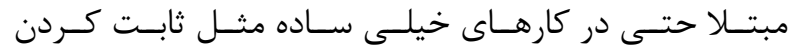

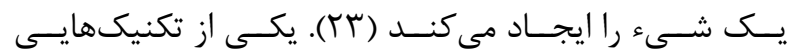

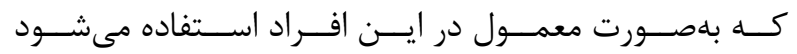

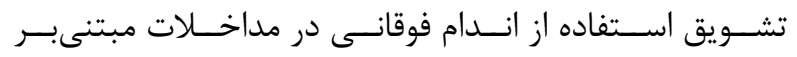

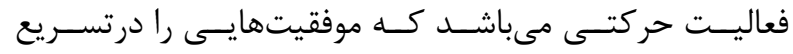

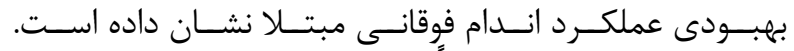

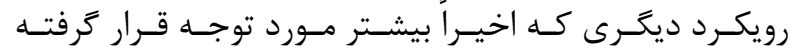

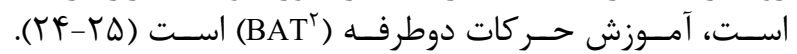

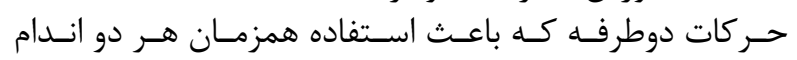

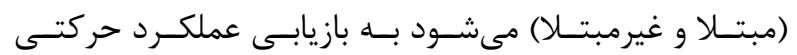

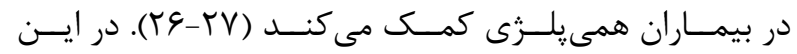

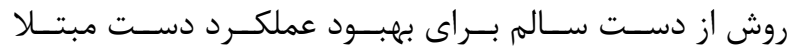

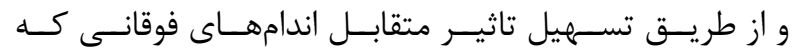

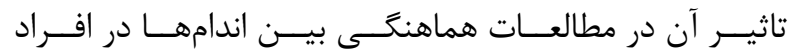

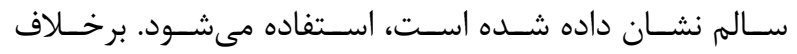

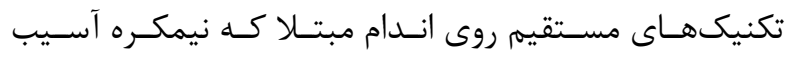

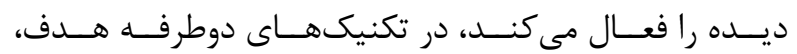

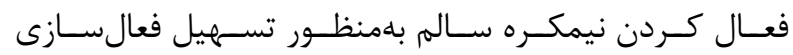

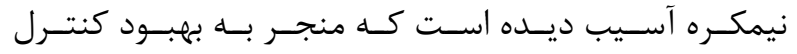

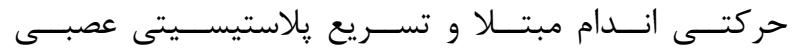

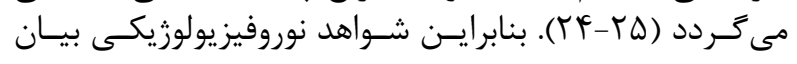

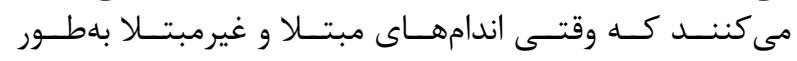

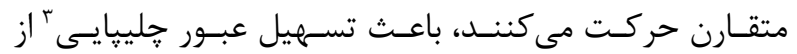

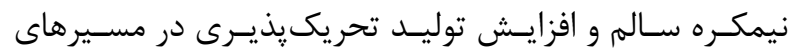

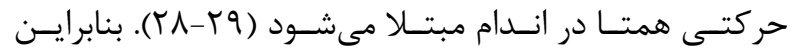

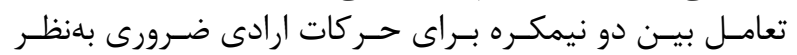

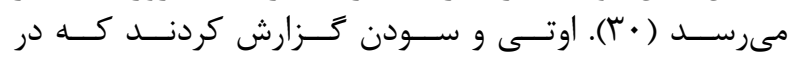

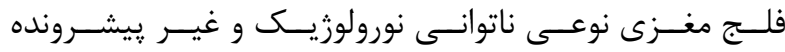

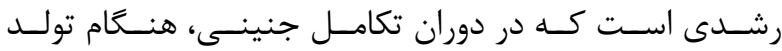

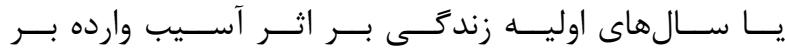

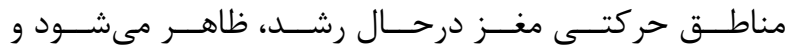

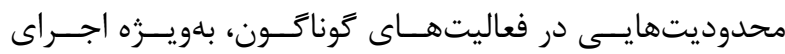

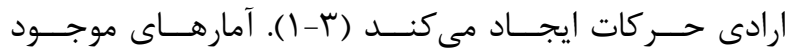

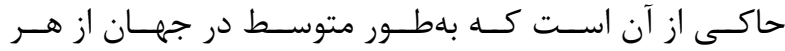

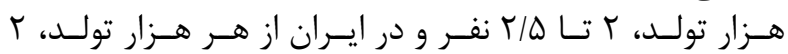

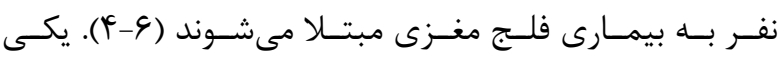

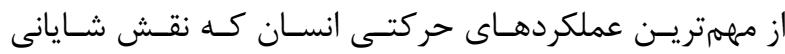

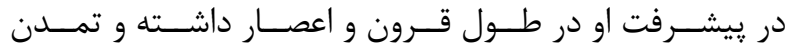

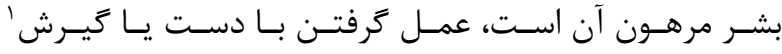

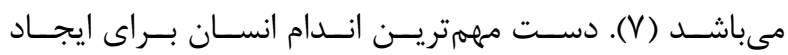

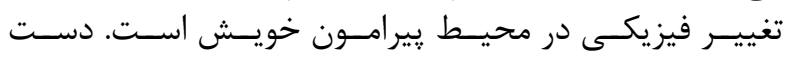

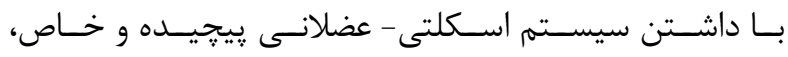

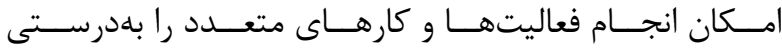

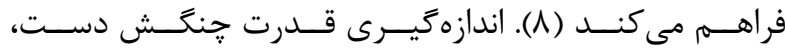

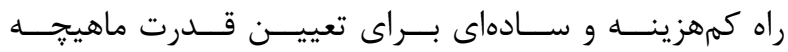

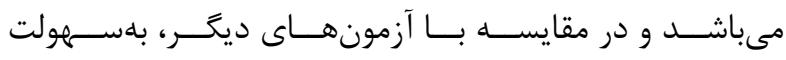

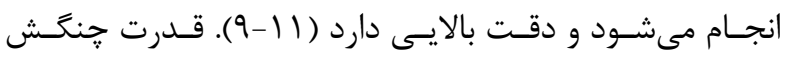

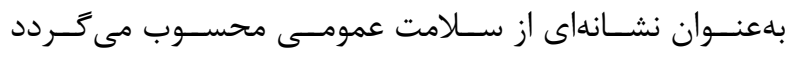

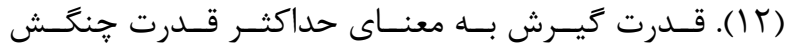

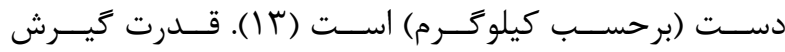

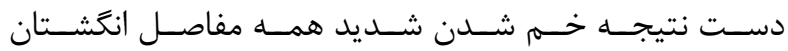

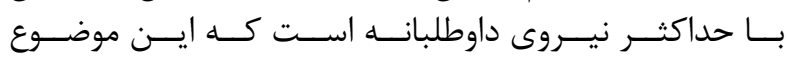

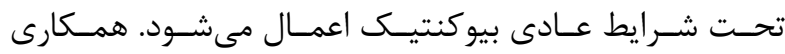

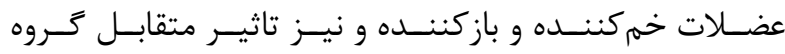

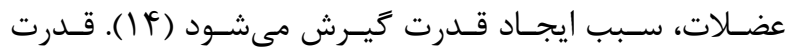

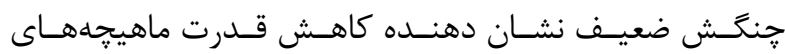

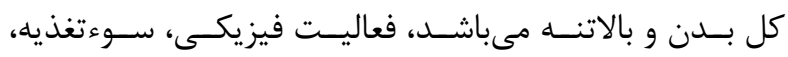

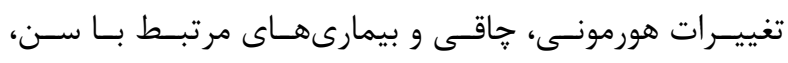

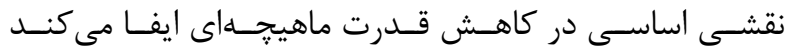

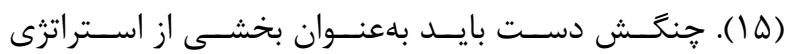

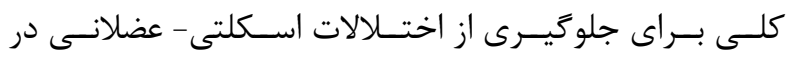

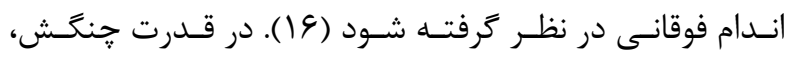

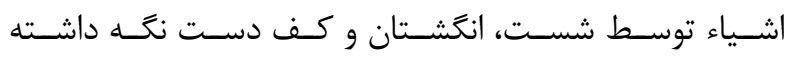

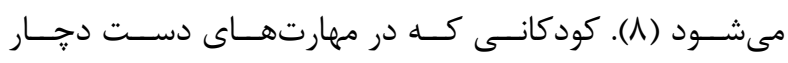

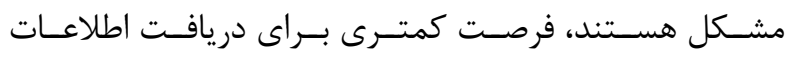

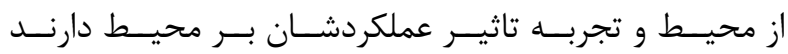

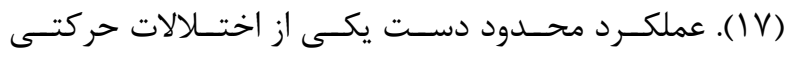

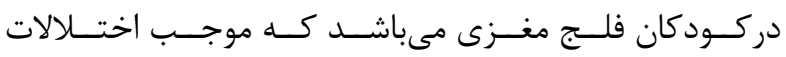

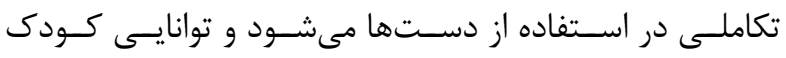

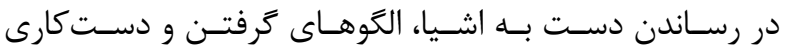




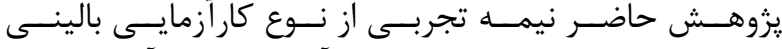

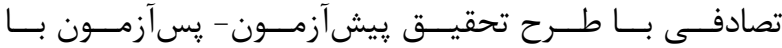

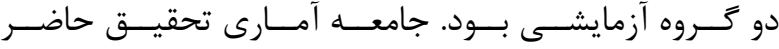

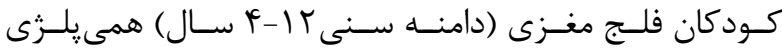

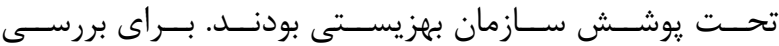

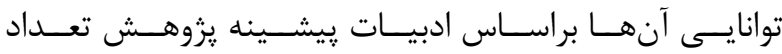

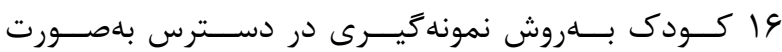

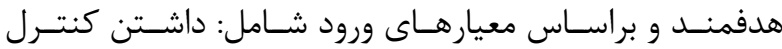

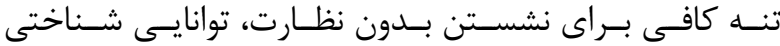

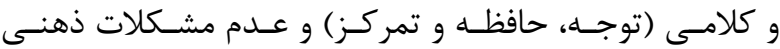

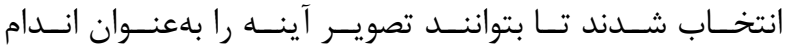

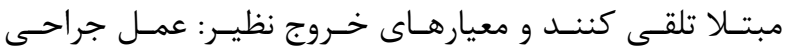

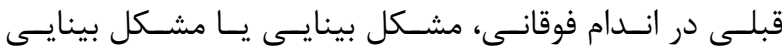

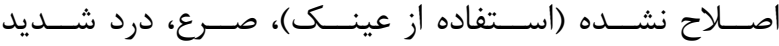

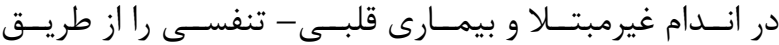

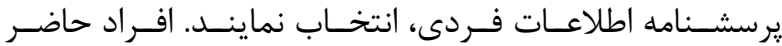

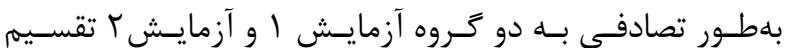

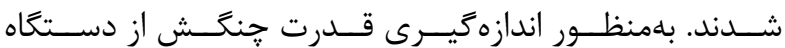

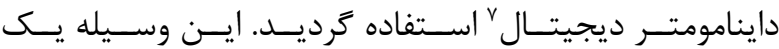

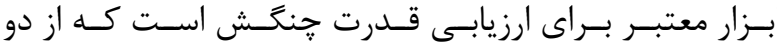

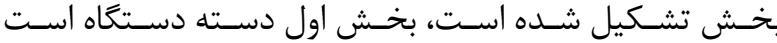

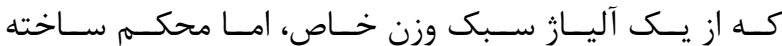

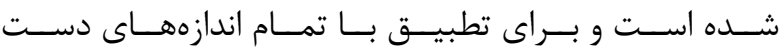

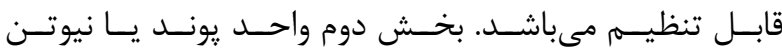

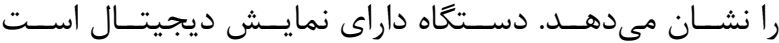

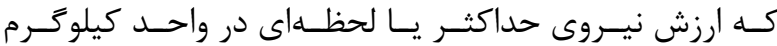

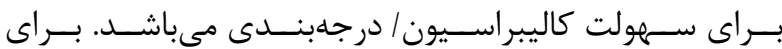

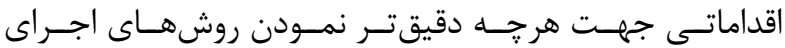

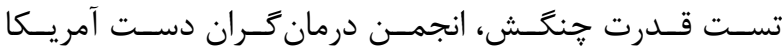

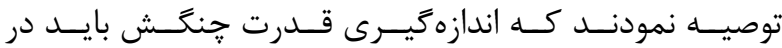

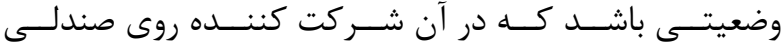

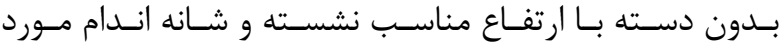

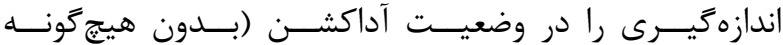

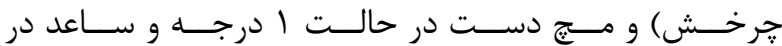

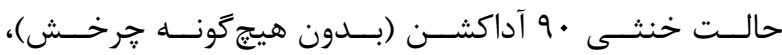

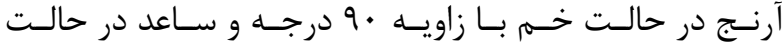

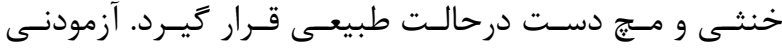

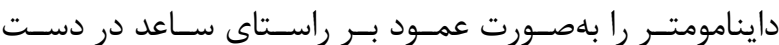

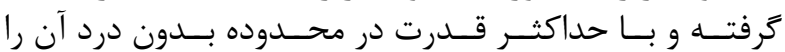

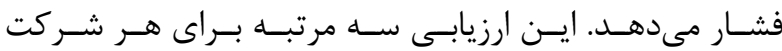

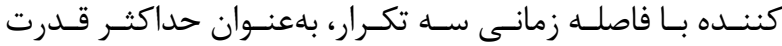

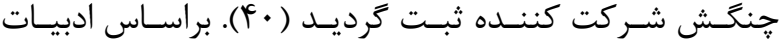

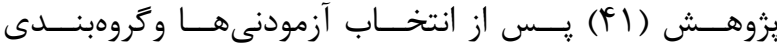

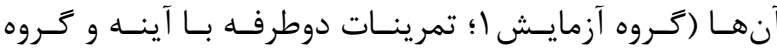

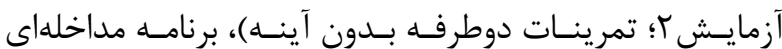

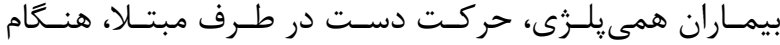

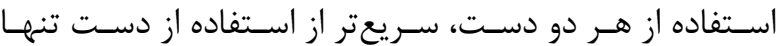

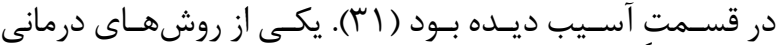

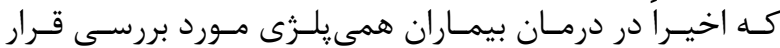

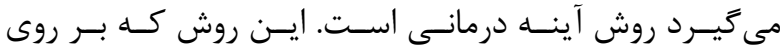

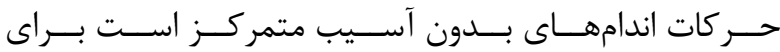

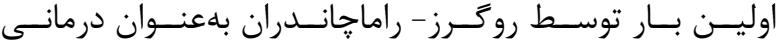

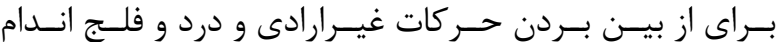

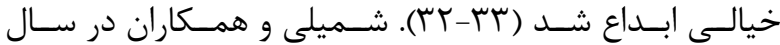

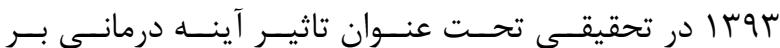

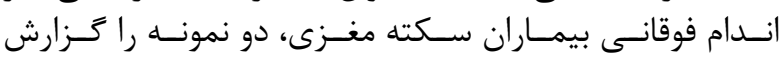

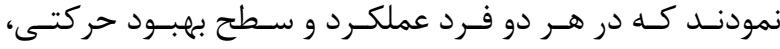

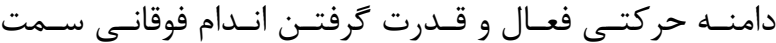

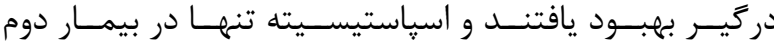

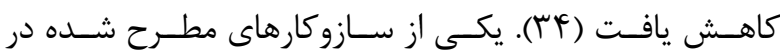

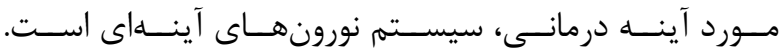

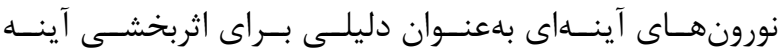

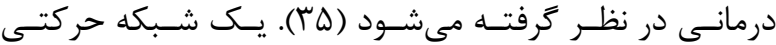

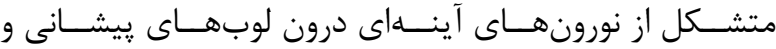

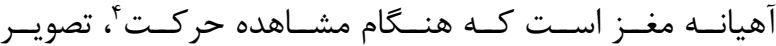

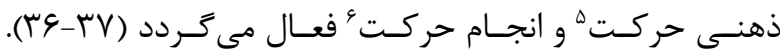

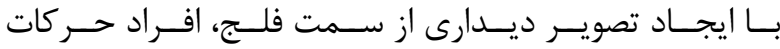

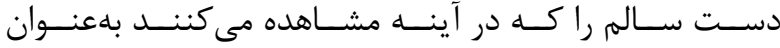

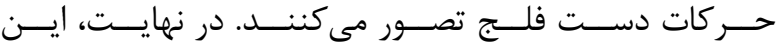

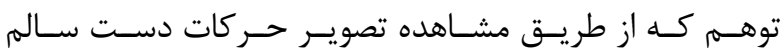

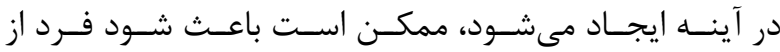

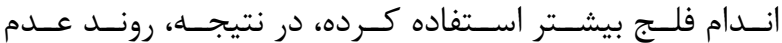

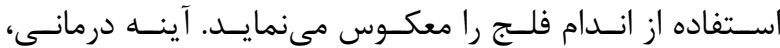

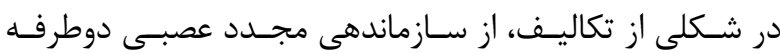

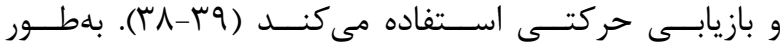

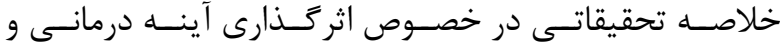

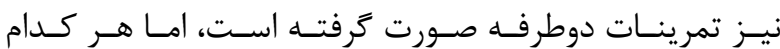

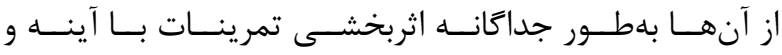

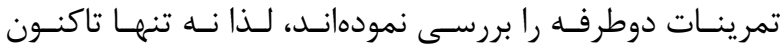

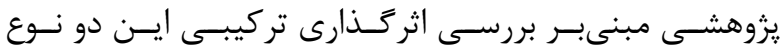

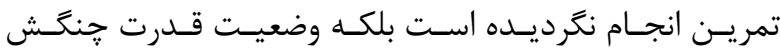

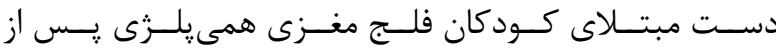

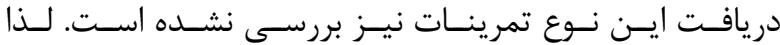

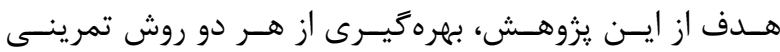

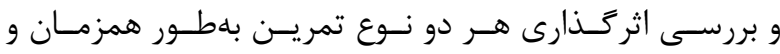

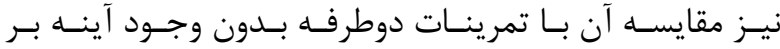

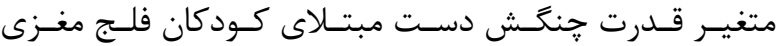

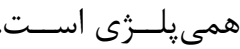

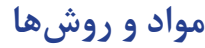

\footnotetext{
${ }^{4}$ Action Observation

${ }^{5}$ Motor Imagery
}

\footnotetext{
${ }^{6}$ Motor Execution

${ }^{7}$ MIE medical research LTD
} 


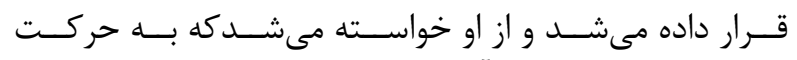

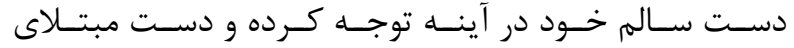

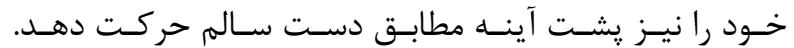

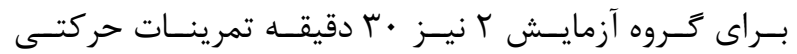

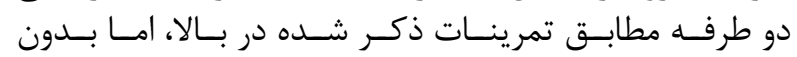

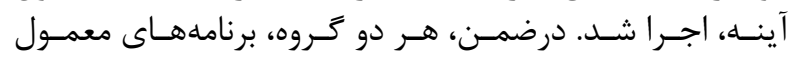

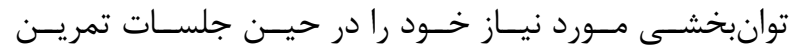

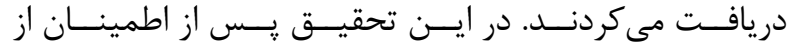

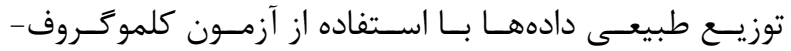

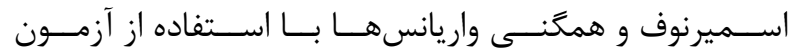

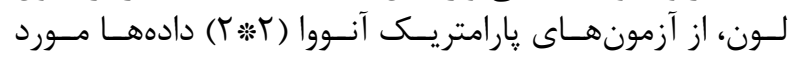

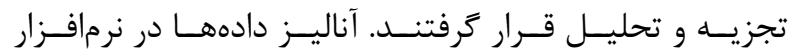

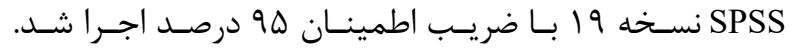

عافتهها

اطلاعـات توصيفـى مربـوط بـه مشـــصات فـردى آزمودنىهــا

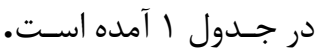

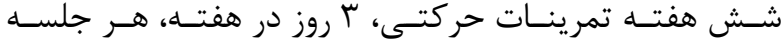

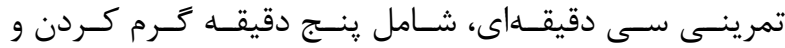

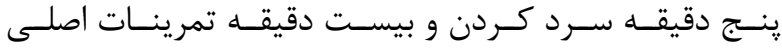

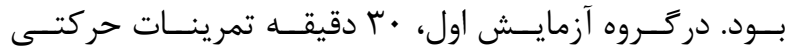

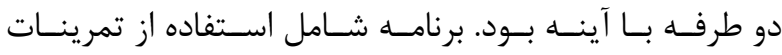

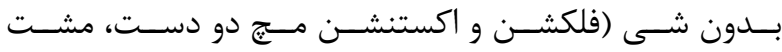

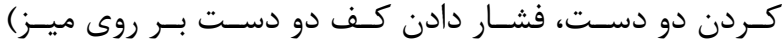

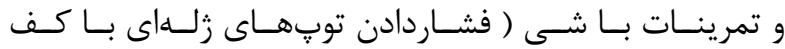

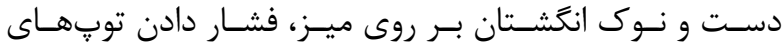

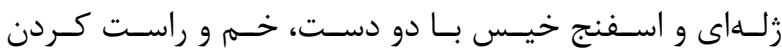

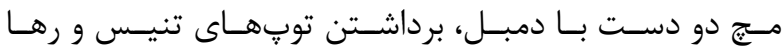

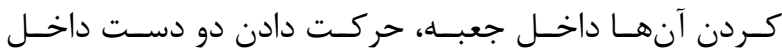

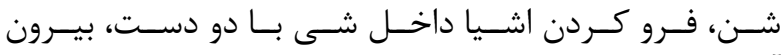

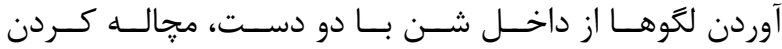

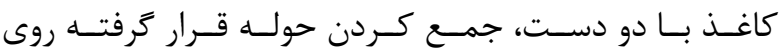

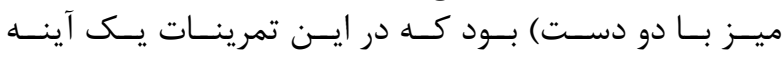

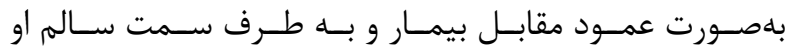

\begin{tabular}{|c|c|c|}
\hline تعداد & سن (برحسب سال) ميانكين\ انحراف معيار & كروه \\
\hline$\Lambda$ & $\Delta /{ }^{\mu} V \pm 1 / q r$ & آزمايش 1 \\
\hline$\Lambda$ & $\varphi / V \Delta \pm 1 / \% \Lambda$ & آزمايش r \\
\hline
\end{tabular}

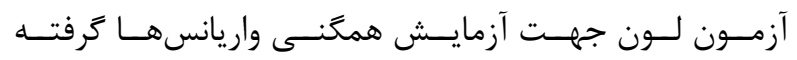

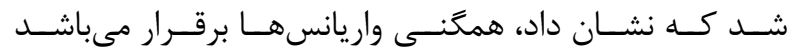

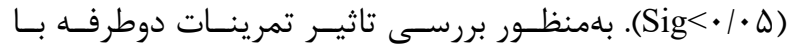

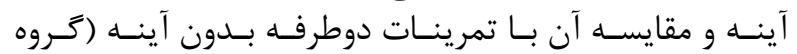

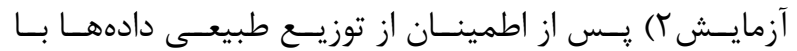

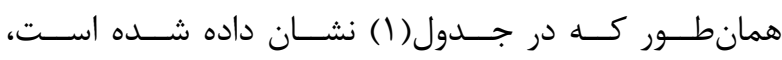

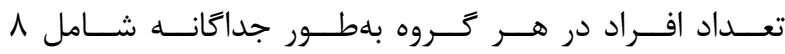

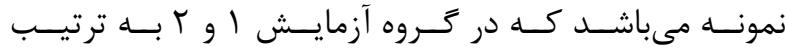

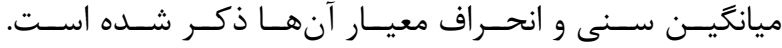

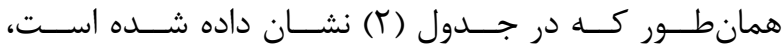

\begin{tabular}{|c|c|c|c|c|}
\hline Sig & Df2 & Df1 & $\mathbf{F}$ & فاكتورهاى اندازهَيرى شده \\
\hline$\cdot 19$ & 14 & 1 & • & قدرت جِنَش \\
\hline
\end{tabular}

جدول r- بررسى همَنى واريانسها (آزمون لون) در هر دو كروه

\begin{tabular}{|c|c|c|c|c|c|}
\hline سطح معنى دارى & Z & ميانگين 土 انحراف معيار & زمان & تروه & فاكتورهاى اندازهَيرى شده \\
\hline .19 & 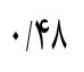 & $r / \boldsymbol{c} \cdot \pm \cdot \mid \Lambda r$ & يَيشآزمون & \multirow{2}{*}{ زمايش 1} & \multirow{4}{*}{ قدرت קنَش } \\
\hline$\cdot 19$ & $\cdot / N T^{\mu}$ & $N / \& V \pm \cdot \mid M \Lambda$ & 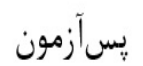 & & \\
\hline$\cdot 19$ & $\cdot \mid \Delta \Delta$ & $r / T^{\mu} \pm \cdot 19 \mu$ & بيشآزمون & \multirow{2}{*}{ آزمايش r } & \\
\hline$\cdot \pi$ & $\cdot|f|$ & $r / Y \Delta \pm \cdot / V F$ & يسآزمون & & \\
\hline
\end{tabular}




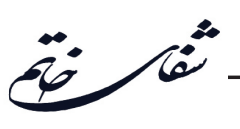

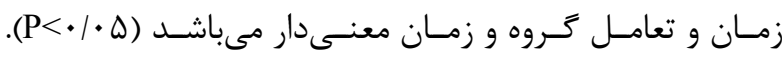

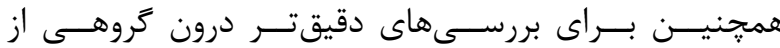

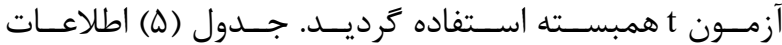

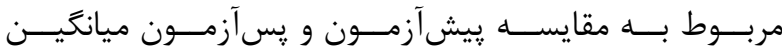

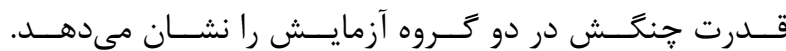

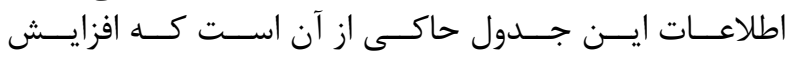

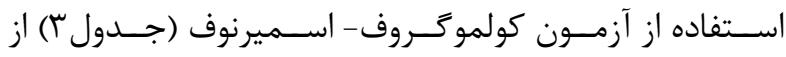

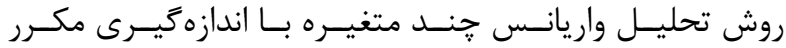

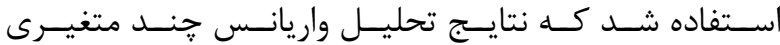

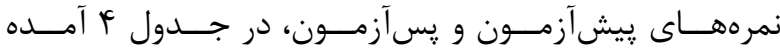

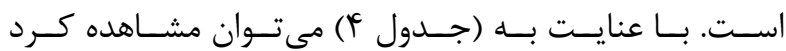

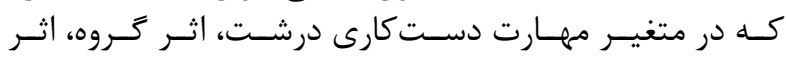

\begin{tabular}{|c|c|c|c|c|c|}
\hline مجذور جزئى اتا & سطح معنى دارى & $\mathbf{F}$ & درجة آزادى & مقايسةُ كرووما & متغير \\
\hline$\cdot / 1$ &.$/ . .1$ & $\Delta 91 \cdot V$ & 1 & كروهها (آزمايش ا، آزمايش؟) & \\
\hline$\cdot 19$ & $\cdot / . .1$ & $V \& 1 / 9 \Delta$ & 1 & زمان (ييشآزمون- يسآزمون) & قدرت جنَش \\
\hline$\therefore / 9$ & $.1 . .1$ & FT. 199 & 1 & تعامل زمان گروهو & \\
\hline
\end{tabular}

\begin{tabular}{|c|c|c|c|c|c|}
\hline Sig & DF & t & يسآزمون MESD & يششآزمون MESD & كروه \\
\hline$\cdot 1 \cdot \cdot 1$ & V & $-r \Delta / \mathcal{F} \Lambda$ & $\Lambda / \mathcal{V} \pm \pm \cdot \mid \Lambda \Lambda$ & $r / \boldsymbol{T} \cdot \pm \cdot \mid \Lambda \mu$ & آزمايش 1 \\
\hline$\cdot|\cdot|$ & V & $-1 \cdot / V F$ & $r / r \Delta \pm \cdot / V \varphi$ & r/TrE • & آزمايش r \\
\hline
\end{tabular}

جدول هـ - نتايج آزمون t همبسته براى قدرت حنتش در دو كروه آزمايش

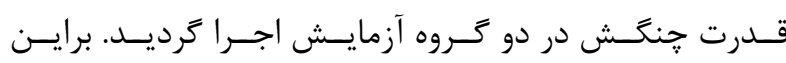

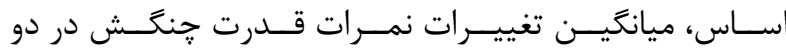

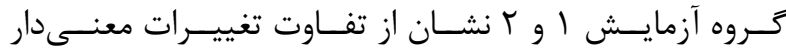

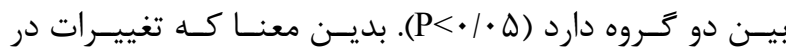

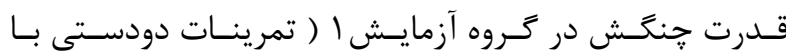

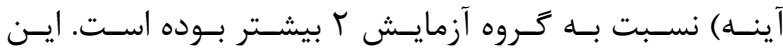

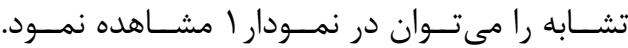
بحث و نتيجهَيرى

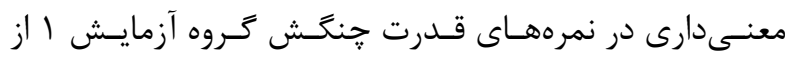

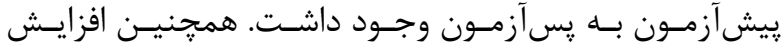

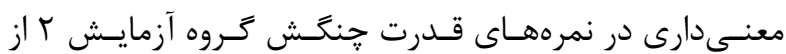

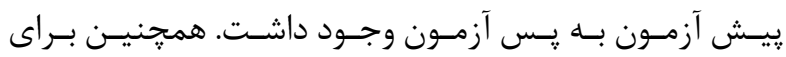

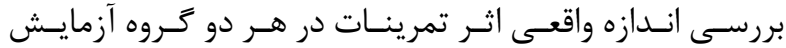

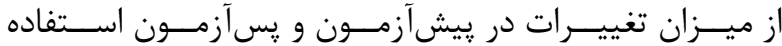

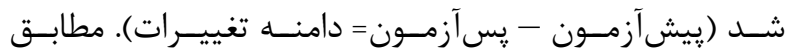

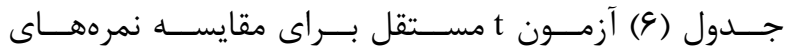

\begin{tabular}{|c|c|c|c|c|}
\hline Sig & 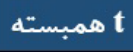 & ميانكَين تغييرات در كروه آزمايش ץ & ميانكَين تغييرات در كروه آزمايش 1 & متغير \\
\hline$\cdot / . .1$ & $r \cdot|Q|$ & . 195 & G/TV & 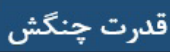 \\
\hline
\end{tabular}

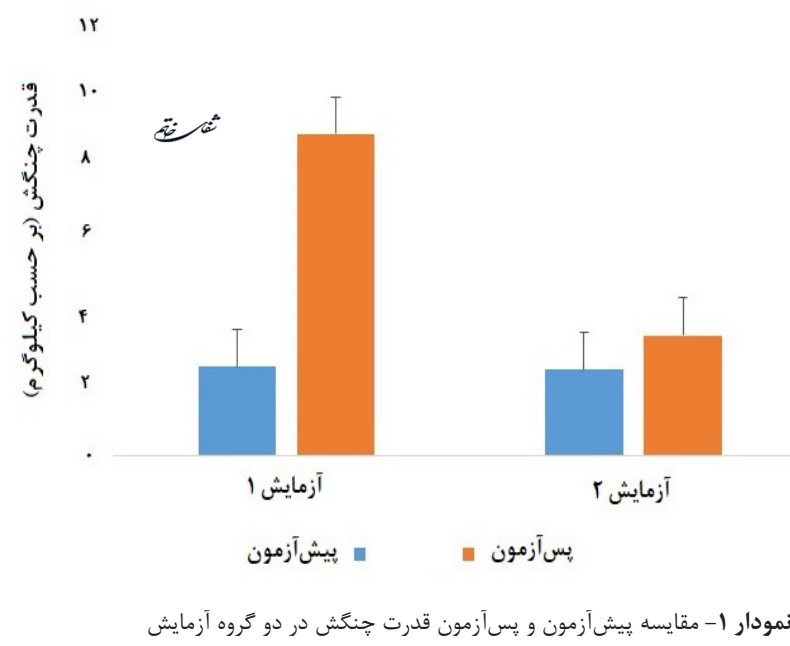




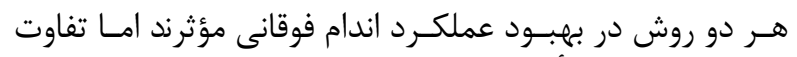

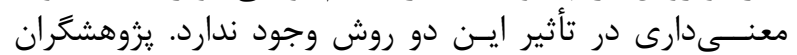

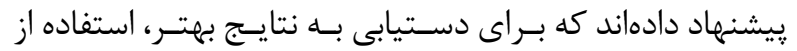

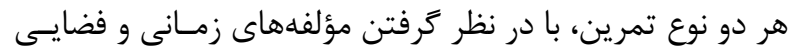

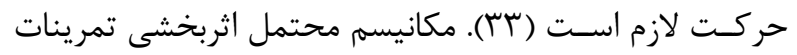

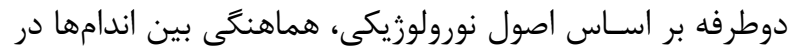

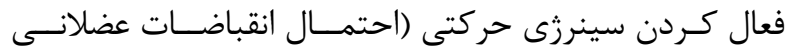

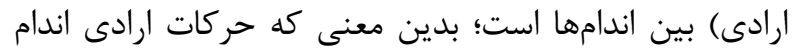

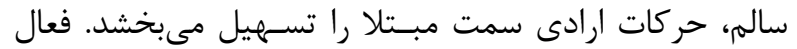

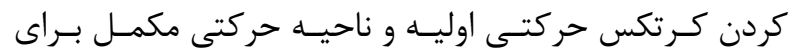

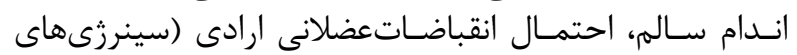

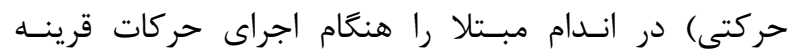

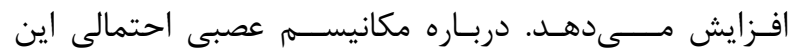

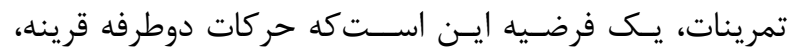

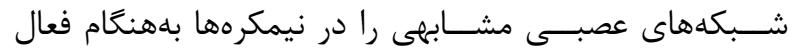

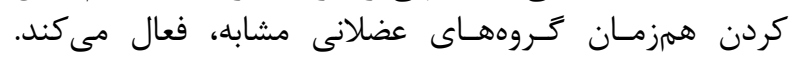

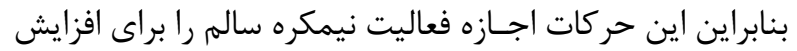

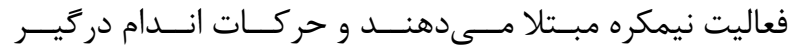

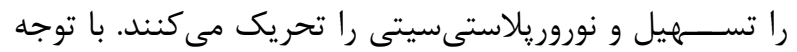

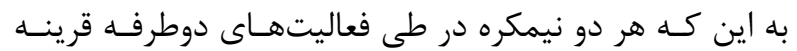

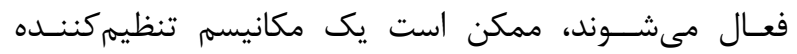

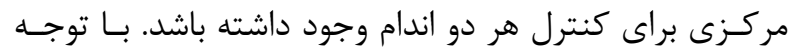

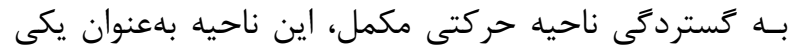

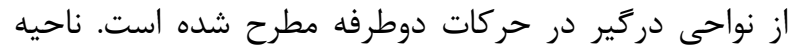

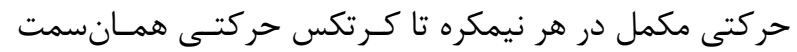

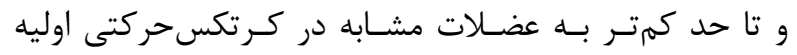

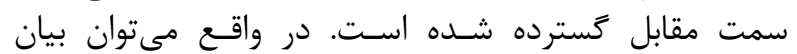

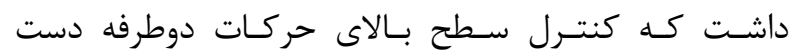

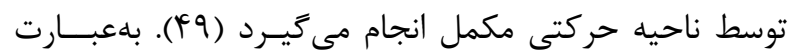

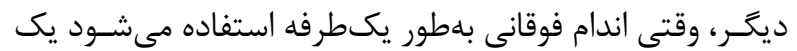

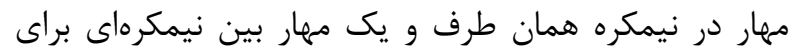

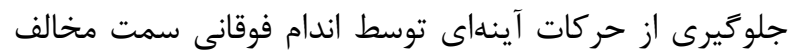

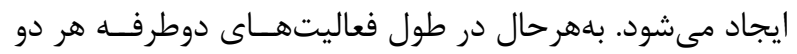

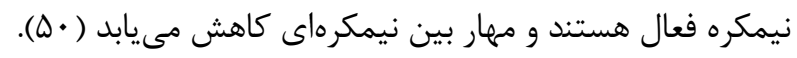

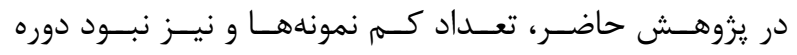

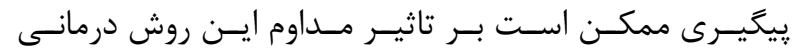

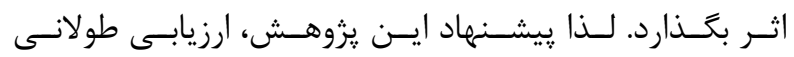

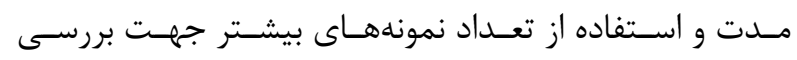

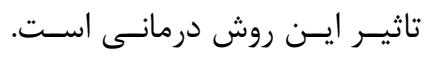

1. Bosanquet, M., Copeland L, Ware R, Boyd R. A systematic review of tests to predict cerebral palsy in young children. Developmental Medicine \& Child Neurology, 2013. 55(5): P. 418-26.

2. Oskoui, M., CoutinhoF, Dykemanj,jetteN, Pringsheim T. An update on the prevalence of cerebral palsy: a

${ }^{8}$ Feltham

${ }^{9}$ Reach

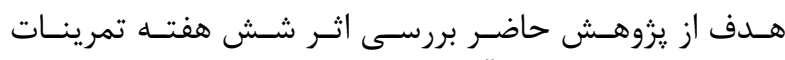

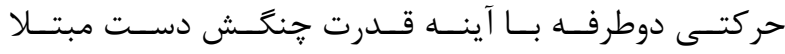

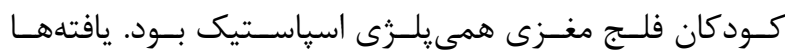

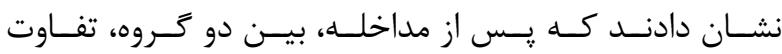

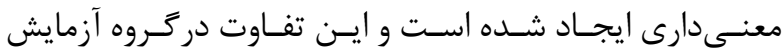

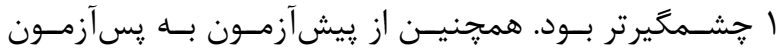

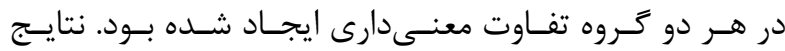

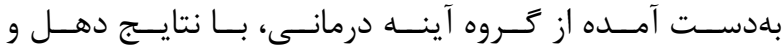

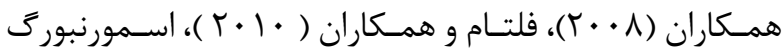

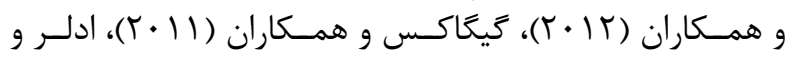

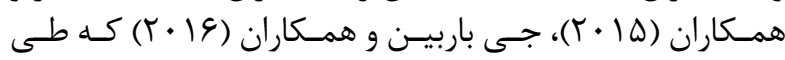

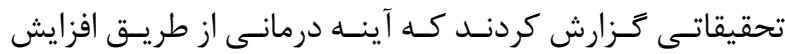

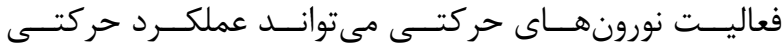

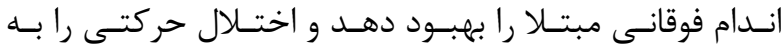

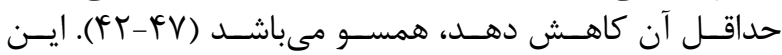

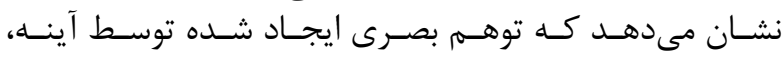

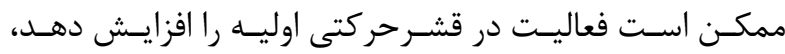

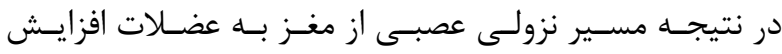

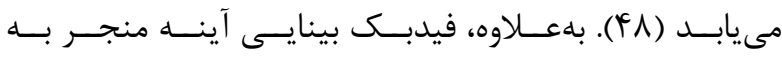

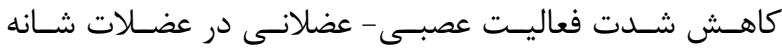

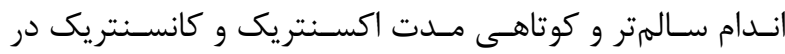

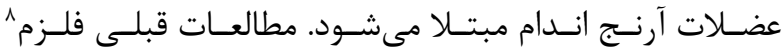

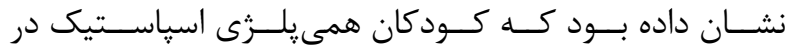

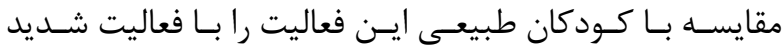

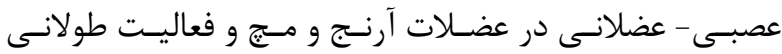

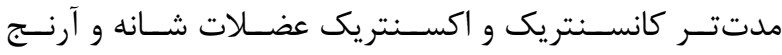

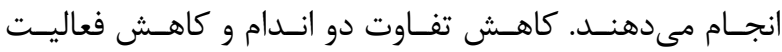

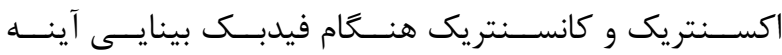

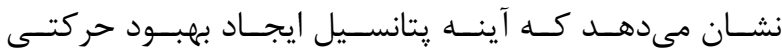

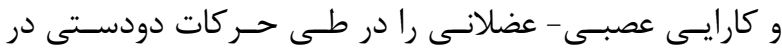

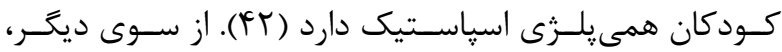

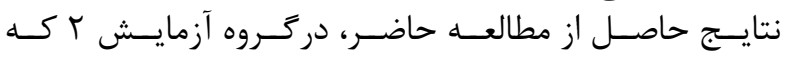

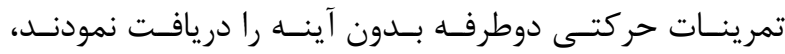

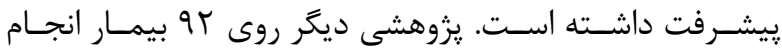

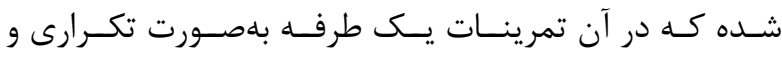

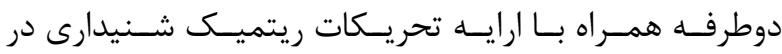

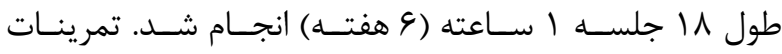

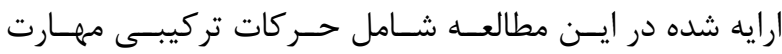

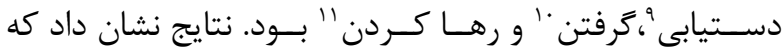

منابع systematic review and meta-analysis. Developmental Medicine \& Child Neurology, 2013. 55(6): P. 509-19.

3. Richards, C.L, Malouin F. Malouin, Cerebral palsy: definition, assessment and rehabilitation. Handbook of clinical neurology, 2013. 111: P. 183-95.

4. Rogers, B. Feeding method and health

${ }^{10}$ Grasp

${ }^{11}$ Release 


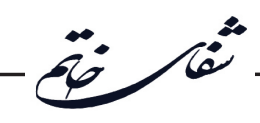

outcomes of children with cerebral palsy. The Journal of pediatrics, 2004. 145(2): P. S28-S32.

5. Linden, P, Siebens A. Siebens, Dysphagia: predicting laryngeal penetration. Archives of physical medicine and rehabilitation, 1983. 64(6): P. 281-84.

6. Sankar, C, Mundkur N. Mundkur, Cerebral palsydefinition, classification, etiology and early diagnosis. The Indian Journal of Pediatrics, 2005. 72(10): P. 865-68.

7. Levangie, P.K. and C.C. Norkin, Joint structure and function: a comprehensive analysis. 2011.

8. Hashemi nezhad N, Choobineh A, Mohammadian M. Comparison of grip and pinch strengths of adults among five cities of Iran. Journal of School of Public Health and Institute of Public Health Research, 2014. 11(3): P. 65-81.

9. Murabito JM, Rong j, Lunetta KL, Huan T, Lim H, Zhao Q. Cross-sectional relations of whole-blood mi RNA expression levels and hand grip strength in a community sample. Aging cell, 2017. 16(4): P. 888-94.

10. Soury S, HABIBI E, Hasan Zadeh A. Measuring factors affecting grip strength base on ASHT (American society of hand therapists. 2, 2015. 10(4): P. 719-28.

11. Jaber R, Hewson DJ, Duchene j. Duchêne, Design and validation of the Grip-ball for measurement of hand grip strength. Medical engineering \& physics, 2012. 34(9): P. 1356-61.

12. Shim Jh, Roh SY, Kin Js, Lee DC, Ki SH, Yang Jw. Normative measurements of grip and pinch strengths of $21 \mathrm{st}$ century Korean population. Archives of plastic surgery, 2013. 40(1): P. 52.

13. Ramlagan S, Peltzer K, Phaswana-Mafuya N. Hand grip strength and associated factors in noninstitutionalised men and women 50 years and older in South Africa. BMC research notes, 2014. 7(1): P. 1-7.

14. Tufts Brown johns Hopkins. Hand Grip Strength Protocol. Tufts University Nutrition Collaborative, 2003.

15. Granic A, Davies K, Martin-Ruiz C, jagger C, Kirkwood TBL, von Zglinicki T. Grip strength and inflammatory biomarker profiles in very old adults. Age and ageing, 2017. 46(6): P. 976-82.

16. McDowell T.W, Winer BM, Welcome DE, Warren C, Dong RG. Effects of handle size and shape on measured grip strength. International Journal of Industrial Ergonomics, 2012. 42(2): P. 199-05.

17. Exner C.E. Development of hand skills. Occupational therapy for children, 2001. 5: p. 304-55.

18. Sadeghi moghadam R, lajvardi L. Investigating the effects of wrist Kinesio Taping on hand function of children with spastic diplegic cerebral palsy. Modern Rehabilitation, 2012. 6(1).

19. Pfeifer LL, Santos TR, Silva DB, Panuncio Pinto MP, Caldas CA, Santos Jl. Hand function in the play behavior of children with cerebral palsy. Scandinavian journal of occupational therapy, 2014. 21(4): P. 241-50.

20.MazzoneS, SerafiniA,IosaM.Functionaltapingapplied to upper limb of children with hemiplegic cerebral palsy: a pilot study. Neuropediatrics, 2011. 42(06): P. 249-53.

21. Kisner, C., L.A. Colby, and J. Borstad, Therapeutic exercise: foundations and techniques. 6th ed. 2017: Fa Davis. 960.

22. Charles J, Gordon AM. A critical review of constraintinduced movement therapy and forced use in children with hemiplegia. Neural plasticity, 2005. 12(2-3): P. 245-61.

23. DeLuca, S., Intensive Movement Therapy with Casting for Children with Hemiparetic Cerebral Palsy: A Randomised Controlled Trial [dissertation]. Birmingham, The University of Alabama, 2002.

24. Stewart, K.C., J.H. Cauraugh, and J.J. Summers, Bilateral movement training and stroke rehabilitation: a systematic review and meta-analysis. Journal of the neurological sciences, 2006. 244(1-2): P. 89-95.

25. Morris JC, VanWijck F, Joice S. A comparison of bilateral and unilateral upper-limb task training in early poststroke rehabilitation: a randomized controlled trial. Archives of physical medicine and rehabilitation, 2008. 89(7): P. 1237-45.

26. Latimer $\mathrm{CP}$, Keeling $\mathrm{j}$, Lin B. The impact of bilateral therapy on upper limb function after chronic stroke: a systematic review. Disability and rehabilitation, 2010. 32(15): P. 1221-31.

27. Van Delden AEQ, Peper CE, Beek Pj. Unilateral versus bilateral upper limb exercise therapy after stroke: a systematic review. Journal of 
rehabilitation medicine, 2012. 44(2): P. 106-17.

28. Cauraugh JH,Summers jj. Neural plasticity and bilateralmovements: arehabilitation approach forchronic stroke. Progress in neurobiology, 2005. 75(5): P 309-20.

29. Carson, R., Neural pathways mediating bilateral interactions between the upper limbs. Brain Research Reviews, 2005. 49(3): P. 641-62.

30. Ferbert A, Vielhaber S, Meincke U, Buchner H. Transcranial magnetic stimulation in pontine infarction: correlation to degree of paresis. Journal of Neurology, Neurosurgery \& Psychiatry, 1992. 55(4): P. 294-99.

31. PGCE, A.U.B. and D.S.M.P. Chair, Interlimb coupling in children with hemiplegic cerebral palsy during reaching and grasping at speed. Developmental Medicine \& Child Neurology, 1998. 40(6): P. 396-04.

32. Ramachandran, V.S., D. RogersRamachandran, and S. Cobb, Touching the phantom limb. Nature, 1995. 377(6549): P. 489-90.

33. McCabe, C.S., R.C. Haigh, and D.R. Blake, Mirror visual feedback for the treatment of complex regional pain syndrome (type 1). Current Pain and Headache Reports, 2008. 12(2): P. 103-07.

34. Waters, P.M. and A. Van Heest, Spastic hemiplegia of the upper extremity in children. Hand clinics, 1998. 14(1): P. 119-34.

35. Carvalho D, Teixeira S, Lucas M. The mirror neuron system in post-stroke rehabilitation. International archives of medicine, 2013. 6(1): P. 1-7.

36. Rizzolatti, G. and L. Craighero, The mirror-neuron system. Annu. Rev. Neurosci., 2004. 27: P. 169-92.

37. Filimon F,Nelson Jd, Sereno MI. Human cortical representations for reaching: mirror neurons for execution, observation, and imagery. Neuroimage, 2007. 37(4): P. 1315-28.

38. Thieme H,Mehrholz j, Pohl M, Behrens j, Dohle C. Mirror therapy for improving motor function after stroke. Stroke, 2013. 44(1): P. e1-e2.

39. Narayan Arya,Pandian S. Effect of task-based mirror therapy on motor recovery of the upper extremity in chronic stroke patients: A pilot study. Topics in Stroke Rehabilitation, 2013. 20(3): P. 210-17.

40. Chao E, Corney W. Focus in the normal and abnormal hand. Orthopaedic Research 2000. 12: P. 202-11.

41. Farzamfar, P., A. Heirani, and M. Sedighi, The effect of motor training in mirror therapy on gross motor skills of the affected hand in children with hemiplegia. Iranian Rehabilitation Journal, 2017. 15(3): P. 243-48.

42. Dohle C,Pullen j, Nakaten A, Kust j, Rietz C, Karbe H. Mirrortherapypromotesrecovery fromseverehemiparesis: a randomized controlled trial. Neurorehabilitation and neural repair, 2008. 23(3): P. 209-17.

43. Feltham $\mathrm{M}$ G, Ledebt $\mathrm{A}$, Deconinck $\mathrm{F} \mathrm{J}$, Savelsbergh G J. Mirror visual feedback induces lower neuromuscular activity in children with spastic hemiparetic cerebral palsy. Research in developmental disabilities, 2010. 31(6): P. 1525-35.

44. Smorenburg A R P, Ledebt A, Deconinck F J A, Savelsbergh G J P. Matching accuracy in hemiparetic cerebral palsy during unimanual and bimanual movements with (mirror) visual feedback. Research in developmental disabilities, 2012. 33(6): P. 2088-98.

45. Adler $\mathrm{C}$, Berweck $\mathrm{S}$, Lidzba $\mathrm{K}$, Becher $\mathrm{T}$, Staudt M. Mirror movements in unilateral spastic cerebral palsy: specific negative impact on bimanual activities of daily living. European journal of paediatric neurology, 2015. 19(5): P. 504-09.

46. Gygax, M.J., P. Schneider, and C.J. Newman, Mirror therapy in children with hemiplegia: a pilot study. Developmental Medicine \& Child Neurology, 2011. 53(5): P. 473-76.

47. J Barbin, V Seetha, Jm, Casillas, j Paysant, D Perennou. The effects of mirror therapy on pain and motor control of phantom limb in amputees: a systematic review. Annals of physical and rehabilitation medicine, 2016. 59(4): P. 270-75.

48. Fukumura K, Sugawara K, Tanabe S, Ushiba j, Tomita Y. Influence of mirror therapy on human motor cortex. International Journal of Neuroscience, 2007. 117(7): P. 1039-48.

49. Ansari, N. and S. Naqdi, Rehabilitation techniques for treating stroke. 2010, Tehran, Arjomand. P. 295-6.

50. Bütefisch C, Hummelsheim H, Denzler $\mathrm{P}$, Mauritz KH. Repetitive training of isolated movements improves the outcome of motor rehabilitation of the centrally paretic hand. Journal of the neurological sciences, 1995. 130(1): P. 59-68. 